\title{
Cybervictimisation and depressive symptoms among adolescents: A moderated mediation model
}

\author{
María Teresa Chamizo-Nieto ${ }^{1}$ (D) . Lourdes Rey ${ }^{1}$ (D
}

Accepted: 18 October 2021

(c) The Author(s) 2021

\begin{abstract}
Recently, there has been an increase in the problem of cyberbullying all over the world, which implies important negative consequences for adolescent victims' mental health, such as depression. There are few research studies that analyse the role of gratitude and cognitive coping strategies for the consequences of cybervictimisation. To date, there are few studies examining the influence of both these resources in a cyberbullying context. Therefore, the objectives of this research were to analyse the mediating role of maladaptive cognitive emotion regulation strategies (CERS) in the relationship between cybervictimisation and depressive symptoms, and to explore the potential moderating role of gratitude among the study variables. A sample of 1156 students between 12 and 18 years (54.33\% females) filled out self-report questionnaires measuring cybervictimisation (ECIPQ), cognitive coping strategies (CERQ-SA), depressive symptoms (DASS-21) and gratitude (GQ5). The outcomes showed that maladaptive CERS partially mediated the relation between cybervictimisation and depressive symptoms. Furthermore, gratitude buffered the relation between maladaptive CERS and depressive symptoms. These findings provide evidence for the buffering role of gratitude in a more frequent use of maladaptive CERS and depressive symptoms in cybervictimisation. The limitations and implications of the study are discussed.
\end{abstract}

Keywords Gratitude $\cdot$ Cognitive emotion regulation strategies · Coping · Depressive symptoms · Cyberbullying · Cybervictimisation

\section{Cyberbullying and Depression}

Cyberbullying is a phenomenon that has increased during the last decade. This problem is defined as intentional and repeated harm to another person through the usage of electronic devices and online technologies (e.g. computers, smartphones, Internet, and social media) (Smith et al., 2008). In contrast to face-to-face aggression, cyberbullying favours the aggressor's anonymity, as well as the access, dissemination, and permanency in cyberspace of the harmful content, being able to be seen by a wider audience (Deschamps \& McNutt, 2016). This makes it more difficult for victims to defend themselves and may lead to

Lourdes Rey

lrey@uma.es

María Teresa Chamizo-Nieto

mtchamizo@uma.es

1 Faculty of Psychology, University of Málaga, Campus de Teatinos s/n, 29071 Málaga, Spain an important negative impact on their health (Deschamps \& McNutt, 2016).

Although prevalence varies among countries, this phenomenon can be found everywhere. For instance, $21.90 \%$ of 12,372 adolescents aged 14-17 years from seven European countries (including Spain) reported suffering from cybervictimisation in the last 12 months (Athanasiou et al., 2018). Likewise, a more recent study pointed out that one in four European children and adolescents between 7 and 19 years had been victims of cyberbullying during the last year (Sorrentino et al., 2019).

A range of studies has provided evidence on the severe short- and long-term negative consequences on the psychological maladjustment of adolescents involved in cyberbullying situations. Adolescents suffering cybervictimisation tend to report lower levels of self-esteem, life satisfaction, selfconcept, as well as a higher frequency of stress, anxiety, fear of negative assessment, and perceived loneliness (Camerini et al., 2020; Estévez et al., 2019; Zhong et al., 2021). Also, these adolescents have a higher likelihood of suicide ideation and attempt (Hinduja \& Patchin, 2019). Furthermore, 
previous research shows evidence that suffering from cyberbullying is a risk factor that increases a greater likelihood of developing depressive symptoms (e.g. Cole et al., 2016; Li et al., 2018; Zhong et al., 2021). Likewise, cybervictimisation may lead to an increased development and duration of depressive symptoms than when other types of face-to-face aggressions are suffered (Cole et al., 2016). Some researchers have suggested that a higher risk of developing depressive symptoms through suffering cybervictimisation might be explained by the characteristics of cyberbullying, such as the aggressor's anonymity or a large audience and the difficulty in eliminating the harmful content, which may decrease the capacity to control the situation or increase victims' feelings of fear and helplessness (e.g. Cole et al., 2016; Sticca \& Perren, 2013).

Few studies have analysed the underlying mechanisms implied in the relationship between cybervictimisation and depressive symptoms (Li et al., 2018). Nevertheless, some recent findings provide evidence that stressful situations, such as suffering cybervictimisation, may influence cognitive schemes (i.e. on how adolescents perceive the world and themselves), which may lead to the development of depressive symptoms (Li et al., 2018; Zhong et al., 2021). Thus, these researchers' findings suggest that the levels of selfesteem and of psychological insecurity may help to understand the linkage between cybervictimisation and depressive symptoms (Li et al., 2018; Zhong et al., 2021). Despite these findings, further research is necessary to acquire a more comprehensive knowledge of this relationship.

\section{Maladaptive Cognitive Emotion Regulation Strategies as Mediators}

According to the stress and coping theory (Folkman et al., 1986), stressful situations will require an appraisal and a making of decisions to cope with them. In addition, Folkman et al. (1986) pointed out the important effects that coping processes have on health, considering them essential mediators between stressful situations and their short- and long-term consequences on well-being and mental health. Thus, how adolescents cope with a stressful situation, such as cybervictimisation, would have important effects on their psychological adjustment, such as the likelihood of developing depressive symptoms. In this regard, emotion regulation is an essential element in the management of stress, being defined as "extrinsic and intrinsic processes responsible for monitoring, evaluating, and modifying emotional reactions, especially their intensive and temporal features, to accomplish one's goals" (Thompson, 1994, pp. 27-28). Garnefski et al. (2001) distinguished nine cognitive emotion regulation strategies (CERS) that people may utilise to cope with negative or stressful situations. These strategies can be classified as adaptive strategies (i.e. acceptance, putting into perspective, planning, positive reappraisal and positive refocusing) and maladaptive strategies (i.e. rumination, catastrophising, self-blame and other-blame).

Scientific literature shows evidence on the negative consequences on psychological adjustment (e.g. higher levels of negative affect, somatic complaints, and depressive symptoms, as well as lower levels of subjective and psychological well-being, among others), through a more frequent use of maladaptive CERS, such as self-blame or rumination, to cope with stressful situation (e.g. Balzarotti et al., 2016; Ray-Yol et al., 2020; Stikkelbroek et al., 2016; Yan et al., 2015). Moreover, previous findings suggest that adolescents and young people tend to more frequent use of maladaptive CERS to cope with stressful situations and to manage negative emotions derived (Maji et al., 2016; Potard et al., 2021; Rey et al., 2020; Stikkelbroek et al., 2016 ; Yan et al., 2015). As Maji et al. (2016) suggest, a more frequent use of maladaptive strategies may be due to a more negative impact on psychological adjustment in stressful situations, such as peer violence, which lead to greater vulnerability and make an adaptive coping by victims more difficult.

Furthermore, according to Folkman et al.'s stress and coping theory (1986), maladaptive CERS could be variables that help to understand the linkage between cybervictimisation and depressive symptoms. Some studies provide evidence on the mediating role of maladaptive CERS in the relationship between suffering negative experiences and developing depressive symptoms (Stikkelbroek et al., 2016; Yan et al., 2015). In the cyberbullying context, some findings such as those by Rey et al. (2020) suggest that suffering cybervictimisation may lead to an increase of internalising symptoms throughout the use of maladaptive CERS. Nevertheless, further research is necessary to confirm these findings and to look deeper into the role of maladaptive CERS in a cyberbullying context. Therefore, considering the aforementioned, we wanted to further the understanding in this regard. Specifically, we wanted to examine whether the relation between cybervictimisation and depressive symptoms may be mediated by overall maladaptive CERS.

\section{Gratitude as Moderator}

One of the factors that has shown evidence of promoting well-being and a better coping with stressful situations is gratitude. This personal resource is defined as a stable predisposition of being aware of and responding to the good actions of others (McCullough et al., 2002), and "a wider life orientation towards noticing and appreciating the positive in the world" (Wood et al., 2010, p. 891 ). Previous research shows that grateful people tend to report higher levels of life satisfaction, positive affect, 
and well-being, as well as lower levels of psychological maladjustment and distress (e.g., Kaniuka et al., 2020; Portocarrero et al., 2020). Moreover, in stressful situations (for example, being cybervictimised), a greater awareness and appreciation of the positive aspects in life may facilitate a broader and more flexible view of these experiences, reducing the tendency to focus on negative aspects, which may help to have a more adaptive coping with these experiences (e.g. Tong \& Oh, 2021) and may reduce their psychological impact (Bono et al., 2020).

There are several theoretical models that try to explain and understand how gratitude can be a resource in helping to cope with negative experiences and to improve well-being. One of these theoretical conceptions is Fredrickson's broaden-and-build model of positive emotions (1998). According to this model, gratitude may be considered a positive emotion that broadens thought-action repertoires, which may facilitate the building of social and personal resources to promote well-being and health. Moreover, the author suggested that positive emotions could "undo the after effects of negative emotions" (Fredrickson, 1998 , p. 314). Thus, being a grateful person would facilitate more psychological resources, such as more competence or more social support, that would help to face more adaptively stressful situations and to mitigate their negative impact on mental health (e.g. preventing the development of depressive symptoms) (e.g. Lau \& Cheng, 2017). Furthermore, some studies provide evidence on how gratitude helps to mitigate the development of depressive symptoms as a consequence of stressful life events among undergraduates (Deichert et al., 2019) and peer victimisation among female adolescents (Rey et al., 2019).

Another theoretical framework is Wood et al.'s coping hypothesis (2007). This hypothesis suggests that grateful people tend to use more positive and active strategies to cope with stressful situations, favouring a better wellbeing and psychological adjustment. According to this hypothesis, previous studies not only provide evidence that grateful people tend to have a more positive coping style (Sun et al., 2019), but they also use in a lower frequency less-adaptive strategies (e.g. rumination) $(\mathrm{H}$. Liang et al., 2020) to face stressful and negative experiences, what help to prevent the development of internalising problems, such as depressive symptoms (H. Liang et al., 2020; Sun et al., 2019). Despite these findings, few studies have looked deeper into the role of gratitude on coping strategies or negative consequences in stressful situations, especially in a cyberbullying context in which it has hardly been studied. Therefore, we wanted to analyse whether gratitude might buffer the negative impact of cybervictimisation on depressive symptoms via maladaptive CERS.

\section{The Present Study}

Given that depression is the fourth leading cause of disease in adolescents and young population (Vos et al., 2020) and that cybervictimisation is one of the risk factors for developing it (Cole et al., 2016), in this study we wanted to explore in more depth the underlying mechanisms in the linkage between cybervictimisation and depressive symptoms. To do so, we tried to resolve some gaps in the literature on the role that factors such as gratitude and cognitive coping could play in a cyberbullying context. There is a particular lack of studies that have analysed the possible protective role of gratitude in cyberbullying situation. Likewise, further studies are needed to confirm and extend the previous findings of the role of cognitive coping in mental health in stressful situations such as cyberbullying situations. For this, we proposed three objectives in this study. First, we wanted to analyse the associations between the four study variables (i.e. cybervictimisation, maladaptive CERS, depressive symptoms, and gratitude), expecting to find positive relationships between cybervictimisation, maladaptive CERS and depressive symptoms, and negative associations between gratitude and the other study variables, in line with previous research (e.g. Estévez et al., 2019; Kaniuka et al., 2020; Ray-Yol et al., 2020; Rey et al., 2019, 2020). Moreover, following Folkman et al.'s stress and coping theory (1986) and previous evidence about the mediating role of maladaptive CERS in the relationship between stressful situations and internalising symptoms (e.g. Rey et al., 2020; Stikkelbroek et al., 2016), it might be assumed that the more cybervictimisation adolescents suffer, the more frequently maladaptive CERS will be used, which may lead to more depressive symptoms. Hence, in our second aim we examined the mediating role of the total score of maladaptive CERS in the relationship between cybervictimisation and depressive symptoms. In our third objective, we explored whether the direct and indirect relationship between cybervictimisation and depressive symptoms via maladaptive CERS would be much weaker for adolescents with higher levels of gratitude. According to the broaden-and-build model (Fredrickson, 1998) and previous evidence of the buffering role of gratitude on negative impact as a consequence of stressful situations (Deichert et al., 2019; Rey et al., 2019), we wanted to examine whether gratitude may play a moderator role in the relationship between cybervictimisation and depressive symptoms. Likewise, due to the fact that a more frequent use of maladaptive CERS is related to an increase in negative affect and depressive symptoms (Balzarotti et al., 2016; Ray-Yol et al., 2020), it is tentatively assumed that gratitude might have a potential buffering 
role on depressive symptoms in those adolescents who make more frequent use of maladaptive CERS to cope with cybervictimisation. Finally, following the coping hypothesis (Wood et al., 2007) and the role of gratitude in favouring a better coping with difficult situations, decreasing the frequency of the use of less-adaptive strategies (H. Liang et al., 2020; Sun et al., 2019), we wanted to explore whether gratitude could interact with cybervictimisation on maladaptive CERS (see Fig. 1). Therefore, the following hypotheses were proposed:

(1) Positive and significant correlations will be found between cybervictimisation, maladaptive CERS and depressive symptoms; in contrast, these correlations will be negative and significant between gratitude and the other three study variables.

(2) The total score of maladaptive CERS will mediate the linkage between cybervictimisation and depressive symptoms.

(3) Gratitude will moderate the direct and indirect relationships in the cybervictimisation-depressive symptoms linkage via maladaptive CERS.

\section{Method}

\section{Participants}

The total sample of this study was 1225 participants, but the final sample comprised 1156 adolescents $(54.33 \%$ females, one participant did not report their sex) between 12 and 18 years old $\left(M_{a g e}=13.78, S D=1.33\right)$ (three participants did not report their age). The data of 69 participants were eliminated because they did not complete at least $80 \%$ of the items of each questionnaire (this is a requirement of the expectation-maximisation (EM) imputation algorithm). The majority of the sample were Spanish (1122 adolescents, $97.06 \%)$. A total of 12 participants (1.03\%) did not report their nationality. The remaining 23 adolescents (1.91\%) were from African (.26\%), Central and South American (.78\%), Asian (.17\%), and other European (.70\%) countries.
The sample were collected from five education centres in southern Spain. The study grades were 7th $(25.95 \%)$, 8th (27.16\%), 9th (22.32\%), and 10th (24.57\%).

\section{Measures}

\section{Socio-Demographic Data}

Some socio-demographic data, such as sex, age, study grade, and nationality were requested from every participant.

\section{Cybervictimisation}

The European Cyberbullying Intervention Project Questionnaire (ECIPQ) subscale of cyberbullying victimisation was used (Del Rey et al., 2015). This comprises 11 items measuring the frequency of cyberbullying behaviour suffered during the last two months. Each item is answered on a Likert-type format with five options ranging from 0 (never to more) to 4 (more times a week). We used the Spanish version providing good psychometric properties $(\alpha=.97$ for cybervictimisation subscale) (Ortega-Ruiz et al., 2016). In the present study, the reliability for this subscale was .85 .

\section{Gratitude}

The Gratitude Questionnaire (GQ) (McCullough et al., 2002) was used to measure the disposition to be grateful. Adolescents answered each item with a seven-point Likerttype format ranging from 1 (totally disagree) to 7 (totally agree). In this study, we used the five-item Spanish version for adolescents, which had adequate indexes of reliability for this population ( $\alpha=.74$ and $\alpha=.77$ for two different adolescent samples) (Rey et al., 2018). In the current study, the reliability was .77 .

\section{Maladaptive Cognitive Emotion Regulation Strategies}

The Cognitive Emotion Regulation Questionnaire (CERQ) (Garnefski et al., 2002) is a questionnaire comprising 36
Fig. 1 The proposed model of moderated mediation

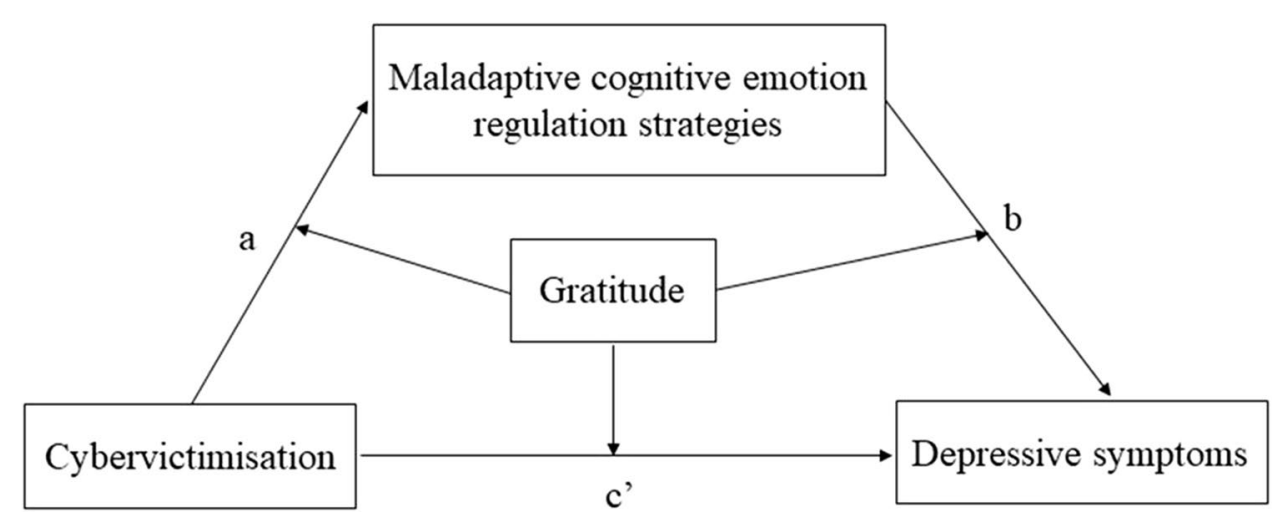


items, which measures nine cognitive strategies to cope with negative events. In this study, the subscales of rumination, catastrophising, self-blame, and other-blame were used. Each item was answered using a five-point Likert scale ranging from 1 (almost never) to 5 (almost always). We used the Spanish version for adolescents, which obtained good psychometric properties (the range of internal consistency indexes for these four subscales was from .62 to .73) (Chamizo-Nieto et al., 2020). In this study, we considered the total punctuation of maladaptive strategies (i.e. first, the scores obtained from the four subscales are added together. Later, that result is divided by four). In this study, the Cronbach's $\alpha$ of the total punctuation of maladaptive strategies was .80 .

\section{Depressive Symptoms}

The Depression, Anxiety and Stress Scales (DASS) subscale of depression was used (Lovibond \& Lovibond, 1995). This subscale comprises seven items assessing the frequency of depressive symptomatology during the last week. Adolescents answered each item using a four-point Likert-type format ranging from 0 (did not apply to me at all) to 3 (applied to me very much or most of the time). The Spanish version, which had adequate reliability index for this subscale $(\alpha=.84)$, was used in this study (Bados et al., 2005). In the present study, the Cronbach's $\alpha$ of this subscale was .90 .

\section{Procedure}

The procedure consisted of filling out a paper-pencil battery of questionnaires by adolescents as part of a larger project, endorsed by the Ethical Committee of University of Malaga (62-2016-H) and in accordance with the Declaration of Helsinki (2013). A total of 12 education centres from the Andalusian region were contacted to participate in this project, with an acceptance rate of $41.67 \%$ (five education centres of the province of Malaga). The directors of these five centres were informed about the purposes and the process of the study and signed a written informed consent. Every centre notified and informed students' parents, who signed a written informed consent (in four centres) or gave a passive consent (i.e. they did not clearly refuse to their adolescent participating in the study) (in one centre).
A written informed consent is not necessary for underage participants. All adolescents collaborated in a voluntary and anonymous way in the study. No personal or identifying data were requested. The battery of questionnaires was administered in class groups in a tutorial action hour. Two members of the research team were introduced, gave the material to every student who could and wanted to participate, provided some instructions, and responded to any questions.

\section{Data Analysis Procedures}

Data analyses were obtained using SPSS v23 and PROCESS macro (Hayes, 2018). First, the missing item values were imputed using the expectation-maximisation (EM) imputation algorithm with SPSS v23, which is an appropriate and widely applied procedure (J. Liang \& Bentler, 2004). Subsequently, Cronbach's $\alpha$, descriptive statistics, and correlation between the study variables were calculated. Secondly, mediation analyses were performed by PROCESS macro (Model 4) (Hayes, 2018) to examine whether the overall of maladaptive CERS mediated the relationship between cybervictimisation and depressive symptoms. Later, we explored the moderating role of gratitude on the relationships among cybervictimisation, maladaptive CERS and depressive symptoms (Model 59) (Hayes, 2018). Age and sex (coded 1 for males and 2 for females) were included as covariates and mean-centred variables were used in all analyses.

\section{Results}

\section{Descriptive Statistics and Correlations}

Cronbach's $\alpha$, means, standard deviations and Pearson's correlations between variables are shown in Table 1. Low levels were reported in cybervictimisation and depressive symptoms, while these levels were moderate and high in maladaptive CERS and gratitude, respectively. Furthermore, we obtained adequate reliability indexes from every measure and significant correlations between variables, as we expected. Cybervictimisation, maladaptive CERS, and depressive symptoms had positive relationships with each other, whereas gratitude was negatively associated with them.
Table 1 Descriptive statistics, internal consistency, and correlations among variables

\begin{tabular}{lllllllll}
\hline & $\alpha$ & Mean & SD & Min-Max & 1 & 2 & 3 & 4 \\
\hline 1. Cybervictimisation & .85 & 0.25 & 0.41 & $0-4$ & - & & \\
2. Maladaptive CERS & .80 & 2.67 & 0.63 & $1-5$ & $.18^{* *}$ & - & \\
3. Depressive symptoms & .90 & 0.70 & 0.77 & $0-3$ & $.35^{* *}$ & $.45^{* *}$ & - & \\
4. Gratitude & .77 & 5.66 & 1.14 & $1-7$ & $-.20^{* *}$ & $-.06^{*}$ & $-.35^{* *}$ & - \\
\hline
\end{tabular}

Notes. $S D=$ Standard deviation, $C E R S=$ Cognitive emotion regulation strategies. $* p<.05, * * p<.01$ 


\section{Mediation Analysis}

As can be seen in Table 2, the mediation analysis showed significant positive effects of cybervictimisation on maladaptive CERS $(\mathrm{B}=.29, p<.001)$ and of maladaptive CERS on depressive symptoms $(\mathrm{B}=.49, p<.001)$. Regarding the influence of cybervictimisation on depressive symptoms, a direct effect $(\mathrm{B}=.48, p<.001)$ and a total effect $(\mathrm{B}=.62$, $p<.001)$ were found. Furthermore, cybervictimisation reported a significant indirect effect on depressive symptoms through maladaptive CERS ( $\mathrm{B}=.14,95 \%$ CI: .10 to .18).

\section{Moderated Mediation Analysis}

We explored whether gratitude had a potential buffering role on the relationship between cybervictimisation, maladaptive CERS and depressive symptoms (see Fig. 1; Model 59) (Hayes, 2018). Therefore, we analysed the moderating role of gratitude on the direct relationship (path: cybervictimisation-depressive symptoms), between cybervictimisation and maladaptive CERS (path a), and between maladaptive CERS and depressive symptoms (path b). Hayes (2018) pointed out that the moderated mediation was established if either paths a or b, or both paths were moderated by gratitude. Moreover, the moderating role of gratitude was analysed in the indirect relationship between cybervictimisation and depressive symptoms via maladaptive CERS (see Table 3).

Neither the direct relationship (cybervictimisation-depressive symptoms) nor path a (cybervictimisation-maladaptive CERS) were moderated by gratitude ( $\mathrm{B}=.06, p>.05 ;$ and $\mathrm{B}=.01, p>.05$, respectively). In contrast, the significant relationship between maladaptive CERS and depressive symptoms (path b) was moderated by gratitude ( $\mathrm{B}=-.05,95 \% \mathrm{CI}:-.095$ to $-.003, p<.05)$. Gratitude was divided into three categories: low (Mean -1 Standard deviation), medium and high (Mean +1 Standard deviation). As can be seen in Fig. 2, the positive relation between maladaptive CERS and depressive symptoms was significant at high $(\mathrm{B}=.42,95 \% \mathrm{CI}: .33$ to $.50, p<.001)$, medium ( $\mathrm{B}=.48,95 \% \mathrm{CI}: .41$ to $.53, p<.001)$ and low $(\mathrm{B}=.53,95 \% \mathrm{CI}: .45$ to $.60, p<.001)$ levels of gratitude. Moreover, the indirect relationship between cybervictimisation and depressive symptoms throughout maladaptive
CERS was moderated by gratitude, this being the indirect effect for adolescents with high (indirect effect $=.12,95 \%$ CI: -.06 to -.20 ), medium (indirect effect $=.13,95 \%$ CI: .09 to .18 ) and low (indirect effect $=.14,95 \%$ CI: -.09 to -.21 ) levels of gratitude.

\section{Discussion}

In the present study, we wanted to deepen our understanding of the mediation and moderation mechanisms implied in the relation between cybervictimisation and depression. To date, this is the first study analysing the mediator role of the overall maladaptive strategies and the moderator role of gratitude in cyberbullying context.

Regarding our first hypothesis, the results obtained confirm it. Likewise, the significant correlations found between the four variables of the study (i.e. cybervictimisation, maladaptive CERS, depressive symptoms, and gratitude) were in line with other studies (e.g. Estévez et al., 2019; Kaniuka et al., 2020; Ray-Yol et al., 2020; Rey et al., 2019, 2020). Therefore, our results suggest that experiencing a highly stressful situation, such as cyberbullying, was related to more depressive symptomatology and a more frequent use of less-adaptive strategies (e.g. rumination, emphasising negative experiences or considering oneself to blame for one's situation), which makes a more adaptive coping strategy for cyberbullying more difficult. Furthermore, we found that adolescents tending to use less-adaptive strategies to face cybervictimisation was associated with a greater development of depressive symptoms. In contrast, reporting more awareness and appreciation of positive things in life was related to suffering fewer cyberbullying behaviours, scoring lower levels of psychological maladjustment, and a lower frequency of choosing less-adaptive strategies that make the management of negative experiences difficult.

The findings of the mediation analysis corroborate our second hypothesis. Thus, the indirect relation between cybervictimisation and depressive symptoms via maladaptive CERS was supported, in accordance with previous research (Rey et al., 2020; Stikkelbroek et al., 2016; Yan et al., 2015), and expanding previous knowledge about the development of internalising problems as consequences of
Table 2 Results of mediation analysis

\begin{tabular}{|c|c|c|c|c|c|c|c|c|c|c|c|}
\hline \multirow[t]{2}{*}{ IV } & \multirow[t]{2}{*}{ M } & \multirow[t]{2}{*}{ DV } & \multicolumn{2}{|l|}{$\mathrm{a}$} & \multicolumn{2}{|l|}{ b } & \multicolumn{2}{|l|}{$c^{\prime}$} & \multicolumn{2}{|l|}{ c-c' } & \multirow{2}{*}{$\begin{array}{l}95 \% \text { CI } \\
\text { lower-upper }\end{array}$} \\
\hline & & & $b$ & $S E$ & $b$ & $S E$ & $b$ & $S E$ & $b$ & $S E$ & \\
\hline $\mathrm{CV}$ & & DS & & & & & $.48 * * *$ & .05 & .14 & .02 & \\
\hline & MCERS & & $.29 * * *$ & .44 & $.49 * * *$ & .03 & & & & & $.097-.182$ \\
\hline
\end{tabular}

Notes. $I V=$ Independent variable, $M=$ Mediator, $D V=$ Dependent variable; $a=$ Effect of IV on M; $b=$ Effect of $\mathrm{M}$ on $\mathrm{DV} ; c^{\prime}=$ Direct effect; $c-c^{\prime}=$ Indirect effect; $C I=$ Confidence interval, $S E=$ Standard error, $C V=$ Cybervictimisation, $M C E R S=$ Maladaptive cognitive emotion regulation strategies, $D S=$ Depressive symptoms. $* * * p<.001$ 
Table 3 Results of moderator mediation analysis
Fig. 2 The moderating role of gratitude on the relation between maladaptive cognitive emotion regulation strategies and depressive symptoms. $* * *$ $p<.001$

\begin{tabular}{|c|c|c|c|c|}
\hline & Coefficient & $S E$ & $\Delta R^{2}$ & $\begin{array}{l}95 \% \text { CI } \\
\text { lower to upper }\end{array}$ \\
\hline $\mathrm{Me}=$ Maladaptive CERS & \multicolumn{4}{|c|}{$R^{2}=.042 ; F(5,1147)=10.160 * * *$} \\
\hline Constant & .027 & .200 & & -.365 to .420 \\
\hline $\operatorname{Sex}(\mathrm{Co})$ & $.112 * *$ & .037 & & .040 to .184 \\
\hline Age (Co) & -.015 & .014 & & -.042 to .013 \\
\hline Cybervictimisation (IV) & $.280 * * *$ & .050 & & .182 to .378 \\
\hline Gratitude (Mo) & -.016 & .016 & & -.048 to .017 \\
\hline Cybervictimisation x Gratitude & .006 & .033 & & -.060 to .071 \\
\hline DV = Depressive symptoms & \multicolumn{4}{|c|}{$R^{2}=.377 ; F(7,1145)=99.123 * * *$} \\
\hline Constant & -.128 & .197 & & -.515 to .259 \\
\hline Sex $(\mathrm{Co})$ & $.191 * * *$ & .036 & & .120 to .263 \\
\hline Age (Co) & $.039 * *$ & .014 & & .012 to .066 \\
\hline Cybervictimisation (IV) & $.418 * * *$ & .050 & & .320 to .516 \\
\hline Maladaptive CERS (Me) & $.471 * * *$ & .029 & & .414 to .528 \\
\hline Gratitude (Mo) & $-.191 * * *$ & .016 & & -.223 to -.160 \\
\hline Cybervictimisation $\mathrm{x}$ Gratitude & .062 & .034 & & -.004 to .128 \\
\hline Maladaptive CERS x Gratitude & $-.049 *$ & .024 & $.002 *$ & -.095 to -.003 \\
\hline
\end{tabular}

Conditional indirect effect of cybervictimisation on depressive symptoms via maladaptive CERS at levels of gratitude

\begin{tabular}{|c|c|c|c|}
\hline & Effect & Boot $S E$ & $\begin{array}{l}\text { Boot } 95 \% \text { CI } \\
\text { lower to upper }\end{array}$ \\
\hline Low gratitude & .144 & .030 & .091 to .207 \\
\hline Medium gratitude & .132 & .025 & .086 to .184 \\
\hline High gratitude & .119 & .035 & .056 to .195 \\
\hline
\end{tabular}

Notes. $M e=$ Mediator, $C o=$ Covariable, $I V=$ Independent variable, $M o=$ Moderator, $D V=$ Dependent variable, $C E R S=$ Cognitive emotion regulation strategies, $S E=$ Standard error, $C I=$ Confidence interval. * $p<.05 ; * * p<.01 ; * * * p<.001$

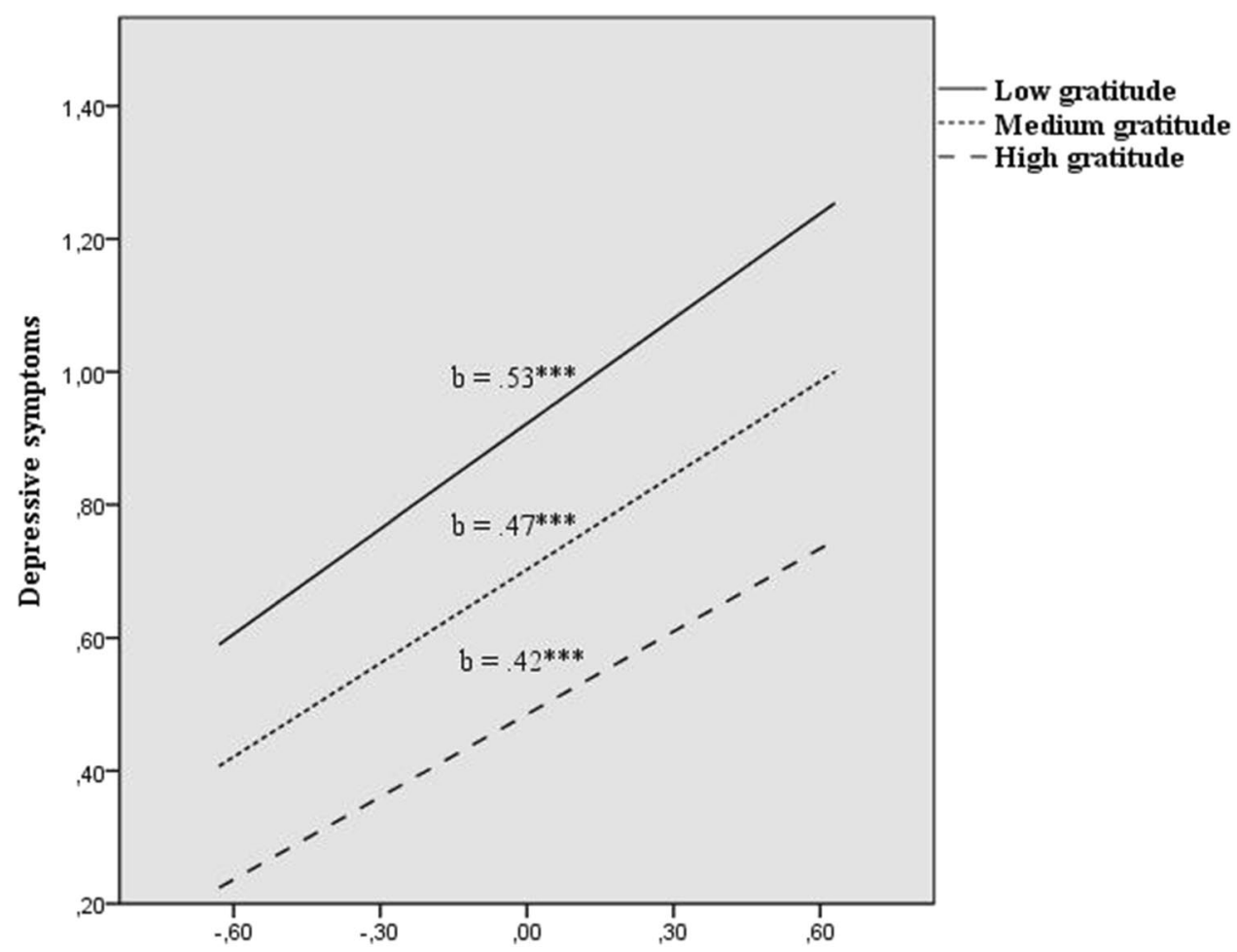

Maladaptive cognitive emotion regulation strategies 
stressful situations. According to Folkman et al.'s stress and coping theory (1986), our findings suggest the importance that maladaptive CERS may have in providing a deeper understanding of the linkage between cybervictimisation and depressive symptoms. Thus, cybervictimisation is a stressful situation that may lead to a greater vulnerability in victims because of its characteristics that differ from face-to-face aggressions, such as a wider audience or a greater permanency of harmful content in the cyberspace (Deschamps \& McNutt, 2016; Sticca \& Perren, 2013). This greater vulnerability in victims (e.g. higher levels of negative emotions or lower levels of self-concept; Camerini et al., 2020; Estévez et al., 2019) may favour the use of maladaptive strategies, such as rumination or self-blame, to manage these situations and their emotions (e.g. Maji et al., 2016; Potard et al., 2021; Rey et al., 2020). Moreover, previous literature shows how a more frequent use of ruminative processes or a greater tendency to blame oneself for negative situations that have occurred may lead to worse psychological adjustment (e.g. developing depressive symptoms or decreasing the levels of well-being) (Balzarotti et al., 2016; Ray-Yol et al., 2020). Therefore, our results provide evidence on the mediating role of maladaptive CERS in the linkage between cybervictimisation and depressive symptoms. Although further research is necessary to deepen the understanding of the linkage between these two complex phenomena (i.e. cybervictimisation and depressive symptoms), this study contributes to showing the importance and consequences on psychological adjustment that the use of specific coping strategies may have in a cyberbullying context.

Considering our third hypothesis, the results of the moderated mediation analysis were partially confirmed. Contrary to previous research (Deichert et al., 2019; Rey et al., 2019), gratitude did not buffer the direct relationship between cybervictimisation and depressive symptoms. Several possible explanations might be considered in this regard. On the one hand, due to the fact that suffering cybervictimisation may lead to more severe negative impact on mental health in comparison to face-to-face aggressions (Deschamps \& McNutt, 2016; Sticca \& Perren, 2013), it is possible that the role of gratitude is not enough by itself, and that other factors and resources have a more direct influence in mitigating the consequences of cybervictimisation on mental health (e.g. perceived social support; Li et al., 2018). On the other hand, considering Deichert et al.'s findings (2019), who report that only an aspect of gratitude (i.e. the appreciation for others) mitigated depressive symptoms caused by stressful events, it might be that only some aspects of gratitude may buffer the direct association between cybervictimisation and depressive symptoms. Therefore, future studies would be necessary to examine in more depth these assumptions.

Nevertheless, gratitude did moderate the indirect relation between cybervictimisation and depressive symptoms via maladaptive CERS. Specifically, our results suggest that those adolescents with higher levels of gratitude will develop fewer depressive symptoms as a consequence of a more frequent use of maladaptive CERS to cope with cybervictimisation. Even though Wood et al.'s coping hypothesis (2007) and previous research (H. Liang et al., 2020; Sun et al., 2019) pointed out grateful people tend to use more adaptive strategies and fewer maladaptive strategies to face stressful situations, we did not find a potential buffering role of gratitude in the linkage between cybervictimisation and maladaptive CERS. One possible explanation for this could be that in a highly stressful situation, such as suffering cybervictimisation, gratitude is not enough to mitigate the tendency to use maladaptive CERS at first, but it does have a moderating role on the negative consequences of their use. According to Fredrickson's broaden-and-build model of positive emotions (1998), gratitude might foster a broader view of these experiences and facilitate the building of resources, and it could also 'undo the after effects of negative emotions' (Fredrickson, 1998, p. 314). So, once some strategies have been used to manage the negative impact as a result of cybervictimisation, a greater awareness and appreciation of positive aspects of life might help to consider other resources to cope with this situation in a more adaptive way, preventing more severe consequences (e.g. the development of depressive symptoms). Thus, our findings provide some evidence of the potential protective role of gratitude on psychological adjustment in adolescents suffering cybervictimisation. Nevertheless, further research is necessary to analyse and corroborate these aspects, as well as to be able to deepen the understanding of the role of gratitude in cyberbullying context.

\section{Limitations}

The current study presents several limitations that should be considered in the interpretation of the findings. Since our sample was collected from a specific geographical area of Spain, the generalisation of our results to adolescents belonging to other regions and countries is restricted. Likewise, we examined some variables implied in the relation between cybervictimisation and depressive symptoms in a non-clinical adolescent sample. Therefore, it would be interesting if these findings could be confirmed in a more heterogeneous sample spanning other regions and countries. Given that this study was designed in a cross-sectional way, performing longitudinal studies would be necessary to confirm whether this moderated mediation model should be maintained over months or even years and go in more depth into the knowledge of the relationship between suffering cybervictimisation and the development of depression in adolescents. Furthermore, future studies could consider examining the influence and interactions of other personal, 
social, and family factors (e.g. personal resources, social support, or socio-economic status, among others), which help one to understand the mechanisms implied, improving the prevention of negative consequences of cyberbullying. Finally, we utilised self-report measures to assess the study variables. Although these instruments reported good reliability indexes, a response bias might have occurred. Therefore, using other informants (e.g. family or teachers), in addition to other measures and assessment methods could help one to understand and broaden the complexity of understanding cyberbullying.

\section{Implications}

Even though this study has some limitations, our findings have important implications. From a theoretical standpoint, the results of this study extend our limited understanding of the complex relation between cyberbullying and depression, delving into mechanisms implied in fostering the development of depressive symptoms by those suffering cybervictimisation and which personal resources or strengths help to buffer that impact. Moreover, the analysis of the combined usage of maladaptive CERS will allow one to advance to a more realistic and complete understanding of coping strategies in cyberbullying contexts and their consequences, taking into account that people do not use one single strategy to manage difficult situations and considering what Garnefski et al. (2001) pointed out about the importance of examining the combination of various strategies to study depression.

From a practical standpoint, these findings point to the necessity of including training of more adaptive strategies in education programmes, which facilitate a better coping in adolescents, mitigating the negative consequences caused by suffering from cyberbullying behaviours. In addition, these outcomes highlight the beneficial consequences on psychology adjustment of being a grateful person in this population, especially in those adolescents who report more difficulty in managing cybervictimisation. Therefore, the development of gratitude in combination with the training of coping strategies may improve prevention programmes for cyberbullying. Since not everyone applies the same tools nor has a good development of adaptive strategies, receiving and expressing gratitude may help to reduce and buffer the psychological distress in those adolescents who tend to use less-adaptive strategies to cope with this problem.

\section{Conclusion}

Taking into account the negative impact of cyberbullying on adolescents' psychological adjustment, and the necessity for delving into the mechanisms involved between cybervictimisation and depression, this study has presented evidence about the protective role of some personal resources. Our findings suggest the importance of developing and training strategies in education programmes to facilitate a better coping with aggressions suffered in cyberspace and through electronic devices. Thus, the negative impact could be buffered and mitigated, improving adolescents' health through a better management of this stressful situation. Moreover, this study provides evidence about the potential protective and buffering role of the development of gratitude against the depressive symptoms of suffering cyberaggression. So, future studies are necessary to further investigate the beneficial consequences of being a grateful person in these situations, hitherto a neglected topic, in order to help to complement and to improve the current programmes against cyberbullying.

Code Availability Not applicable.

Authors' Contributions María Teresa Chamizo-Nieto Writing - original draft preparation Lourdes Rey Writing - review and editing, Funding acquisition. All authors have read and agreed to the published version of the manuscript.

Funding Open Access funding provided thanks to the CRUE-CSIC agreement with Springer Nature. This work was supported by University of Málaga, PAIDI Group CTS-1048 (Junta de Andalucía), Junta de Andalucía/FEDER funds (UMA18-FEDERJA-147) and R+D+i project of the Ministry for Science and Innovation in Spain (PID2020-117006RB-I00).

Data Availability The datasets generated during and/or analysed during the current study are available on reasonable request to M.T.ChamizoNieto,mtchamizo@uma.es

\section{Declarations}

Ethics Approval This study was performed in line with the principles of the Declaration of Helsinki. Approval was granted by the Ethics Committee of University of Málaga (62-2016-H).

Consent to Participate In four education centres, written informed consent was obtained from the parents. In one education centre, a passive consent was obtained from the parents (they did not clearly refuse adolescent's participation in the study).

Conflict of Interest The authors declare that they have no conflict of interest.

Open Access This article is licensed under a Creative Commons Attribution 4.0 International License, which permits use, sharing, adaptation, distribution and reproduction in any medium or format, as long as you give appropriate credit to the original author(s) and the source, provide a link to the Creative Commons licence, and indicate if changes were made. The images or other third party material in this article are included in the article's Creative Commons licence, unless indicated otherwise in a credit line to the material. If material is not included in the article's Creative Commons licence and your intended use is not permitted by statutory regulation or exceeds the permitted use, you will need to obtain permission directly from the copyright holder. To view a copy of this licence, visit http://creativecommons.org/licenses/by/4.0/. 


\section{References}

Athanasiou, K., Melegkovits, E., Andrie, E. K., Magoulas, C., Tzavara, C. K., Richardson, C., Greydanus, D., Tsolia, M., \& Tsitsika, A. K. (2018). Cross-national aspects of cyberbullying victimization among 14-17-year-old adolescents across seven European countries. BMC Public Health, 18, 800. https://doi.org/10.1186/ s12889-018-5682-4

Bados, A., Solanas, A., \& Andrés, R. (2005). Psychometric properties of the Spanish version of depression, anxiety and stress scales (DASS). Psicothema, 17(4), 679-683.

Balzarotti, S., Biassoni, F., Villani, D., Prunas, A., \& Velotti, P. (2016). Individual differences in cognitive emotion regulation: Implications for subjective and psychological well-being. Journal of Happiness Studies, 17(1), 125-143. https://doi.org/10.1007/ s10902-014-9587-3

Bono, G., Reil, K., \& Hescox, J. (2020). Stress and wellbeing in urban college students in the U.S. during the COVID-19 pandemic: Can grit and gratitude help? International Journal of Wellbeing, 10(3), 39-57. https://doi.org/10.5502/ijw.v10i3.1331

Camerini, A.-L., Marciano, L., Carrara, A., \& Schulz, P. J. (2020). Cyberbullying perpetration and victimization among children and adolescents: A systematic review of longitudinal studies. Telematics and Informatics, 49, 101362. https://doi.org/10.1016/j.tele. 2020.101362

Chamizo-Nieto, M. T., Rey, L., \& Sánchez-Álvarez, N. (2020). Validation of the spanish version of the cognitive emotion regulation questionnaire in adolescents. Psicothema, 32(1), 153-159. https:// doi.org/10.7334/psicothema2019.156

Cole, D. A., Zelkowitz, R. L., Nick, E., Martin, N. C., Roeder, K. M., Sinclair-McBride, K., \& Spinelli, T. (2016). Longitudinal and incremental relation of Cybervictimization to negative self-cognitions and depressive symptoms in young adolescents. Journal of Abnormal Child Psychology, 44(7), 1321-1332. https://doi.org/ 10.1007/s10802-015-0123-7

Declaration of Helsinki. (2013). Ethical principles for medical research involving human subjects. Journal of the American Medical Association, 310(20), 2191-2194. https://doi.org/10.1001/jama.2013. 281053

Deichert, N. T., Chicken, M. P., \& Hodgman, L. (2019). Appreciation of others buffers the associations of stressful life events with depressive and physical symptoms. Journal of Happiness Studies, 20(4), 1071-1088. https://doi.org/10.1007/ s10902-018-9988-9

Del Rey, R., Casas, J. A., Ortega-Ruiz, R., Schultze-Krumbholz, A., Scheithauer, H., Smith, P., Thompson, F., Barkoukis, V., Tsorbatzoudis, H., Brighi, A., Guarini, A., Pyzalski, J., \& Plichta, P. (2015). Structural validation and cross-cultural robustness of the European cyberbullying intervention project questionnaire. Computers in Human Behavior, 50, 141-147. https://doi.org/10.1016/j. chb.2015.03.065

Deschamps, R., \& McNutt, K. (2016). Cyberbullying: What's the problem? Canadian Public Administration/Administration Publique Du Canada, 59(1), 45-71.

Estévez, E., Estévez, J. F., Segura, L., \& Suárez, C. (2019). The influence of bullying and cyberbullying in the psychological adjustment of victims and aggressors in adolescence. International Journal of Environmental Research and Public Health, 16(12), 2080. https://doi.org/10.3390/ijerph16122080

Folkman, S., Lazarus, R. S., Gruen, R. J., \& DeLongis, A. (1986). Appraisal, coping, health status, and psychological symptoms. Journal of Personality and Social Psychology, 50(3), 571-579. https://doi.org/10.1037/0022-3514.50.3.571

Fredrickson, B. L. (1998). What good are positive emotions? Review of General Psychology, 2(3), 300-319.
Garnefski, N., Kraaij, V., \& Spinhoven, P. (2001). Negative life events, cognitive emotion regulation and emotional problems. Personality and Individual Differences, 30(8), 1311-1327. https://doi.org/10. 1016/S0191-8869(00)00113-6

Garnefski, N., Kraaij, V., \& Spinhoven, P. (2002). Manual for the use of cognitive emotion regulation questionnaire. DATEC.

Hayes, A. F. (2018). Introduction to mediation, moderation, and conditional process analysis. A regression-based approach (2nd ed.). Guilford Press.

Hinduja, S., \& Patchin, J. W. (2019). Connecting adolescent suicide to the severity of bullying and cyberbullying. Journal of School Violence, 18(3), 333-346. https://doi.org/10.1080/15388220. 2018.1492417

Kaniuka, A. R., Rabon, J. K., Brooks, B. D., Sirois, F., Kleiman, E., \& Hirsch, J. K. (2020). Gratitude and suicide risk among college students: Substantiating the protective benefits of being thankful. Journal of American College Health. https://doi.org/10.1080/ 07448481.2019 .1705838

Lau, B. H. P., \& Cheng, C. (2017). Gratitude and coping among familial caregivers of persons with dementia. Aging and Mental Health, 21(4), 445-453. https://doi.org/10.1080/13607863.2015.1114588

Li, Y., Li, D., Li, X., Zhou, Y., Sun, W., Wang, Y., \& Li, J. (2018). Cyber victimization and adolescent depression: The mediating role of psychological insecurity and the moderating role of perceived social support. Children and Youth Services Review, 94, 10-19. https://doi.org/10.1016/j.childyouth.2018.09.027

Liang, J., \& Bentler, P. M. (2004). An EM algorithm for fitting twolevel structural equation models. Psychometrika, 69(1), 101-122. https://doi.org/10.1007/BF02295842

Liang, H., Chen, C., Li, F., Wu, S., Wang, L., Zheng, X., \& Zeng, B. (2020). Mediating effects of peace of mind and rumination on the relationship between gratitude and depression among Chinese university students. Current Psychology, 39, 1430-1437. https:// doi.org/10.1007/s12144-018-9847-1

Lovibond, P. F., \& Lovibond, S. H. (1995). The structure of negative emotional states: Comparison of the depression anxiety stress scales (DASS) with the Beck depression and anxiety inventories. Behaviour Research and Therapy, 33(3), 335-343. https://doi.org/ 10.1016/0005-7967(94)00075-U

Maji, S., Bhattacharya, S., \& Ghosh, D. (2016). Cognitive coping and psychological problems among bullied and non-bullied adolescents. Journal of Psychosocial Research, 11(2), 387.

McCullough, M. E., Emmons, R. A., \& Tsang, J.-A. (2002). The grateful disposition: A conceptual and empirical topography. Journal of Personality and Social Psychology, 82(1), 112-127. https://doi. org/10.1037/0022-3514.82.1.112

Ortega-Ruiz, R., Del Rey, R., \& Casas, J. A. (2016). Evaluar el bullying y el cyberbullying validación española del EBIP-Q y del ECIP$\mathrm{Q}$ [Assessing bullying and cyberbullying: Spanish validation of EBIPQ and ECIPQ]. Psicología Educativa, 22(1), 71-79. https:// doi.org/10.1016/j.pse.2016.01.004

Portocarrero, F. F., Gonzalez, K., \& Ekema-Agbaw, M. (2020). A metaanalytic review of the relationship between dispositional gratitude and well-being. Personality and Individual Differences, 164, 110101. https://doi.org/10.1016/j.paid.2020.110101

Potard, C., Kubiszewski, V., Combes, C., Henry, A., Pochon, R., \& Roy, A. (2021). How adolescents cope with bullying at school: Exploring differences between pure victim and bully-victim roles. International Journal of Bullying Prevention. https://doi.org/10. 1007/s42380-021-00095-6

Ray-Yol, E., Ülbe, S., Temel, M., \& Altan-Atalay, A. (2020). Interpersonal emotion regulation strategies: Can they function differently under certain conditions? Current Psychology, 4. https://doi.org/ 10.1007/s12144-020-00771-8

Rey, L., Sánchez-Álvarez, N., \& Extremera, N. (2018). Spanish gratitude questionnaire: Psychometric properties in adolescents and 
relationships with negative and positive psychological outcomes. Personality and Individual Differences, 135, 173-175. https://doi. org/10.1016/j.paid.2018.07.005

Rey, L., Quintana-Orts, C., Mérida-López, S., \& Extremera, N. (2019). Being bullied at school: Gratitude as potential protective factor for suicide risk in adolescents. Frontiers in Psychology, 10, 662. https://doi.org/10.3389/fpsyg.2019.00662

Rey, L., Neto, F., \& Extremera, N. (2020). Cyberbullying victimization and somatic complaints: A prospective examination of cognitive emotion regulation strategies as mediators. International Journal of Clinical and Health Psychology, 20(2), 135-139. https://doi. org/10.1016/j.ijchp.2020.03.003

Smith, P. K., Mahdavi, J., Carvalho, M., Fisher, S., Russell, S., \& Tippett, N. (2008). Cyberbullying: Its nature and impact in secondary school pupils. Journal of Child Psychology and Psychiatry, and Allied Disciplines, 49(4), 376-385. https://doi.org/10.1111/j. 1469-7610.2007.01846.x

Sorrentino, A., Baldry, A. C., Farrington, D. P., \& Blaya, C. (2019). Epidemiology of cyberbullying across Europe: Differences between countries and genders. Educational Sciences: Theory and Practice, 19(2), 74-91. https://doi.org/10.12738/estp.2019.2.005

Sticca, F., \& Perren, S. (2013). Is cyberbullying worse than traditional bullying? Examining the differential roles of medium, publicity, and anonymity for the perceived severity of bullying. Journal of Youth and Adolescence, 42(5), 739-750. https://doi.org/10.1007/ s10964-012-9867-3

Stikkelbroek, Y., Bodden, D. H. M., Kleinjan, M., Reijnders, M., \& van Baar, A. L. (2016). Adolescent depression and negative life events, the mediating role of cognitive emotion regulation. PLoS One, 11(8), 1-16. https://doi.org/10.1371/journal.pone.0161062

Sun, P., Sun, Y., Jiang, H., Jia, R., \& Li, Z. (2019). Gratitude and problem behaviors in adolescents: The mediating roles of positive and negative coping styles. Frontiers in Psychology, 10, 1547. https:// doi.org/10.3389/fpsyg.2019.01547

Thompson, R. A. (1994). Emotion regulation: A theme in search of definition. Monographs of the Society for Research in Child
Development, 59(2-3), 25-52. https://doi.org/10.1111/j.15405834.1994.tb01276.x

Tong, E. M. W., \& Oh, V. Y. S. (2021). Gratitude and adaptive coping among Chinese Singaporeans during the beginning of the COVID-19 pandemic. Frontiers in Psychiatry, 11, 628937. https:// doi.org/10.3389/fpsyt.2020.628937

Vos, T., Lim, S. S., Abbafati, C., Abbas, K. M., Abbasi, M., Abbasifard, M., Abbasi-Kangevari, M., Abbastabar, H., Abd-Allah, F., Abdelalim, A., Abdollahi, M., Abdollahpour, I., Abolhassani, H., Aboyans, V., Abrams, E. M., Abreu, L. G., Abrigo, M. R. M., Abu-Raddad, L. J., Abushouk, A. I., ... Murray, C. J. L. (2020). Global burden of 369 diseases and injuries in 204 countries and territories, 1990-2019: A systematic analysis for the global burden of disease study 2019. The Lancet, 396(10258), 1204-1222. https://doi.org/10.1016/S0140-6736(20)30925-9

Wood, A. M., Joseph, S., \& Linley, P. A. (2007). Coping style as a psychological resource of grateful people. Journal of Social and Clinical Psychology, 26(9), 1076-1093. https://doi.org/10.1521/ jscp.2007.26.9.1076

Wood, A. M., Froh, J. J., \& Geraghty, A. W. A. (2010). Gratitude and well-being: A review and theoretical integration. Clinical Psychology Review, 30(7), 890-905. https://doi.org/10.1016/j.cpr. 2010.03.005

Yan, J., Kong, T., He, Y., Mcwhinnie, C. M., Yao, S., \& Xiao, J. (2015). Daily hassles and depression in individuals with cognitive vulnerability to depression: The mediating role of maladaptive cognitive emotion regulation strategies. Nordic Psychology, 67(1), 87-100. https://doi.org/10.1080/19012276.2014.957717

Zhong, M., Huang, X., Huebner, E. S., \& Tian, L. (2021). Association between bullying victimization and depressive symptoms in children: The mediating role of self-esteem. Journal of Affective Disorders, 294, 322-328. https://doi.org/10.1016/j.jad.2021.07.016

Publisher's note Springer Nature remains neutral with regard to jurisdictional claims in published maps and institutional affiliations. 